\title{
Response of anthocyanin biosynthesis to light by strand-specific transcriptome and miRNA analysis in Capsicum annuum
}

Yan Zhou', Muhammad Ali Mumtaz' , Yonghao Zhang ${ }^{2}$, Zhuang Yang ${ }^{1}$, Yuanyuan Hao' ', Huangying Shu', Jie Zhu', Wenlong Bao', Shanhan Cheng ${ }^{1}$, Guopeng Zhu' and Zhiwei Wang ${ }^{1 *}$

\begin{abstract}
Background: Anthocyanins have distinct biological functions in plant coloring, plant defense against strong light, UV irradiation, and pathogen infection. Aromatic hydroxyl groups and ortho-dihydroxyl groups in anthocyanins are able to inhibit free-radical chain reactions and hydroxyl radicals. Thus, anthocyanins play an antioxidative role by removing various types of ROS. Pepper is one of the solanaceous vegetables with the largest cultivation area in China. The purple-fruited pepper is rich in anthocyanins, which not only increases the ornamental nature of the pepper fruit but also benefits the human body. In this experiment, light-induced regulatory pathways and related specific regulators of anthocyanin biosynthesis were examined through integrative transcriptomic and metabolomic analysis.
\end{abstract}

Results: Results revealed that delphinium 3-O-glucoside significantly accumulated in light exposed surface of pepper fruit after $48 \mathrm{~h}$ as compared to shaded surface. Furthermore, through strand-specific sequencing technology, 1341 differentially expressed genes, 172 differentially expressed IncRNAs, 8 differentially expressed circRNAs, and 28 differentially expressed miRNAs were identified significantly different among both surfaces. The flavonoid synthesis pathway was significantly enriched by KEGG analysis including SHT (XM_016684802.1), AT-like (XM_016704776.1), CCOAOMT (XM_016698340.1,XM_016698341.1), CHI (XM_016697794.1,XM_016697793.1), CHS2 (XM_016718139.1), CHS1B (XM_016710598.1),CYP98A2-like (XM_016688489.1),DFR (XM_016705224.1), F3'5'H (XM_016693437.1), F3H (XM_016705025.1), F3'M (XM_016707872.1), LDOX (XM_016712446.1), TCM (XM_016722116.1) and TCM-like (XM_016722117.1). Most of these significantly enriched flavonoid synthesis pathway genes may be also regulated by IncRNA. Some differentially expressed genes encoding transcription factors were also identified including MYB4like (XM_016725242.1), MYB113-like (XM_016689220.1), MYB308-like (XM_016696983.1,XM_016702244.1), and EGL1 (XM_016711673.1). Three'IncRNA-miRNA-mRNA' regulatory networks with sly-miR5303, stu-miR5303g, stu-miR7997a, and stu-miR7997c were constructed, including 28 differentially expressed mRNAs and 6 differentially expressed IncRNAs.

Conclusion: Possible light regulated anthocyanin biosynthesis and transport genes were identified by transcriptome analysis, and confirmed by qRT-PCR. These results provide important data for further understanding of the anthocyanin metabolism in response to light in pepper.

\footnotetext{
*Correspondence: wangzhiwei@hainanu.edu.cn; zwwang22@163.com

1 Key Laboratory for Quality Regulation of Tropical Horticultural Crops

of Hainan Province/Engineering Research Center of the Ministry

of Education for New Variety Breeding of Tropical Crop, School

of Horticulture, Hainan University, Haikou 570228, China

Full list of author information is available at the end of the article
} original author(s) and the source, provide a link to the Creative Commons licence, and indicate if changes were made. The images or other third party material in this article are included in the article's Creative Commons licence, unless indicated otherwise in a credit line to the material. If material is not included in the article's Creative Commons licence and your intended use is not permitted by statutory regulation or exceeds the permitted use, you will need to obtain permission directly from the copyright holder. To view a copy of this licence, visit http://creativecommons.org/licenses/by/4.0/. The Creative Commons Public Domain Dedication waiver (http://creativeco mmons.org/publicdomain/zero/1.0/) applies to the data made available in this article, unless otherwise stated in a credit line to the data. 
Keywords: Pepper, Anthocyanin, Transcriptome, IncRNA, miRNA, Photosensitivity

\section{Background}

Flavonoids are natural products well known for their health benefits as they are considered essential components in various medicinal, pharmaceutical, and cosmetic applications [1]. Studies have shown that flavonoids such as anthocyanins have antioxidant properties [2], and play an important role in drought tolerance [3], resistance to UV-B damage [4, 5], and pathogens [6]. With the development of society, people have a stronger desire to pursue high-quality natural foods that are beneficial to health [7-9].

Capsicum $\mathrm{L}$. is an important solanaceous vegetable with a large cultivation area globally. Pepper fruit has a broad spectrum of health benefits and a unique spicy taste that makes it an indispensable food ingredient. In addition to being rich in vitamin $\mathrm{C}$, capsaicin, carotenoids, purple pepper also contains numerous secondary metabolites such as anthocyanins [10]. The biosynthesis mechanism of flavonoids is one of the most important metabolic pathways currently being studied [11]. Delphinidin-3-trans-coumaroylrutinoside-5-glucoside is the main anthocyanin in purple pepper. Anthocyanins are synthesized in the cytoplasm and are finally transported by flavonoid transporters to the vacuole for storage [7]. Taking a synthetic pathway of capsicum flavonoids as an example, phenylalanine is first catalyzed by phenylalanine ammonia-lyase to cinnamic acid. It then undergoes a series of enzyme activities, such as cinnamic acid 4-hydroxylase $(\mathrm{C} 4 \mathrm{H})$, 4-coumarin-CoA ligase (4Cl), ketone synthase (CHS), chalcone isomerase $(\mathrm{CHI})$, and flavone 3-hydroxylase $(\mathrm{F} 3 \mathrm{H})$ that catalyzes the synthesis of dihydrokaempferol. Then it is catalyzed by dihydroflavonol 4-reductase (DFR) into leucopelargonidin to form colorless anthocyanins [12, 13]. Further, under the catalysis of anthocyanin synthase (ANS), colored anthocyanins are synthesized and transported by transferase to the vacuole for storage.

In most plants except radish, carrot, and potato, anthocyanin biosynthesis and accumulation are induced by light [1]. The synthesis of anthocyanins is regulated by a variety of transcription factors, such as R2R3MYB [14], basic helix-loop-helix (bHLH) [15], WD40 protein [16, 17], and WRKY [18]. Especially, MBW (MYB-bHLHWDR) [19] protein complex play an important role in the flavonoid synthesis pathway. This study aimed to analyze light-induced anthocyanin synthetic regulation through transcriptome analysis (including mRNA and non-coding RNA). Our results identified anthocyanin biosynthesis and transport genes regulated by light and establish a strong basis for further exploring the molecular mechanism of anthocyanin synthesis and regulation in pepper.

\section{Results}

Changes in fruit color and anthocyanin content after light treatment

After $48 \mathrm{~h}$ of different light treatment, the light-exposed surface had a light purple color (Fig. 1b), while the color of the fruit on the shaded side was still milky white without purple coloration. The total content of anthocyanin were $3.57 \mu \mathrm{g} / \mathrm{g}$ (freeze-dried) in the light treated and $0.523 \mu \mathrm{g} / \mathrm{g}$ (freeze-dried) in the dark treated pepper fruits (Fig. 1h).

\section{Determination of types and contents of anthocyanins by LC-MS/MS}

LC-MS/MS was used to determine and calculate the total anthocyanin content. The total contents of L48 and D48 were $3.3104 \mu \mathrm{g} / \mathrm{g}$ and $0.5686 \mu \mathrm{g} / \mathrm{g}$, respectively (Fig. 2), which is consistent with the results of our determination of total anthocyanin content using the $\mathrm{pH}$ differential method. Delphinidin 3-O-glucoside was determined as the main anthocyanins in pepper fruit. The content of Delphinidin 3-O-glucoside in L48 were $1.11 \mu \mathrm{g} / \mathrm{g}$ and $0.383 \mu \mathrm{g} / \mathrm{g}$ in D48, accounting for 33.53 and $67.41 \%$ of the total content, respectively. Delphinidin 3-O- $\left(6^{\prime}-\right.$ O-malonyl)-beta-D-glucoside, Peonidin3-O-(6-O-malonyl-beta-D-glucoside), and Petunidin 3-O-glucoside were present in L48 and D48 in minor quantity and remained unchanged in different light treatments. Pelargonidin 3-O-galactoside was only present in the D48 $(0.0132 \mu \mathrm{g} / \mathrm{g})$. And Delphinidin 3-O-rutinoside, Cyanidin 3-O-rutinoside, Petunidin 3-O-(6-O-malonyl-beta-Dglucoside), Petunidin 3-O-rutinoside and other 13 anthocyanins were only synthesized in L48. Interestingly, a higher amount of Quercetin 3-O-glucoside was observed in L48 $(26.167 \mu \mathrm{g} / \mathrm{g})$. These results show that light intensity largely affects the synthesis of flavonols.

\section{Mapping and quality analysis of total transcriptome RNA}

To explore the anthocyanins synthesis pathway in purple pepper fruits, six chain-specific RNA libraries (including mRNA, lncRNA, and circRNA) and six small RNA libraries were constructed for the transcriptome analysis of D48 and L48. For strand-specific RNA sequencing, the original readings of these six samples ranged from $10,317.48$ to 1354.71356 million, resulting in RNA-Seq clean bases ranging from 15.04 to $19.88 \mathrm{G}$. The Q20 percentage exceeded $97.88 \%$, the Q30 percentage exceeded 


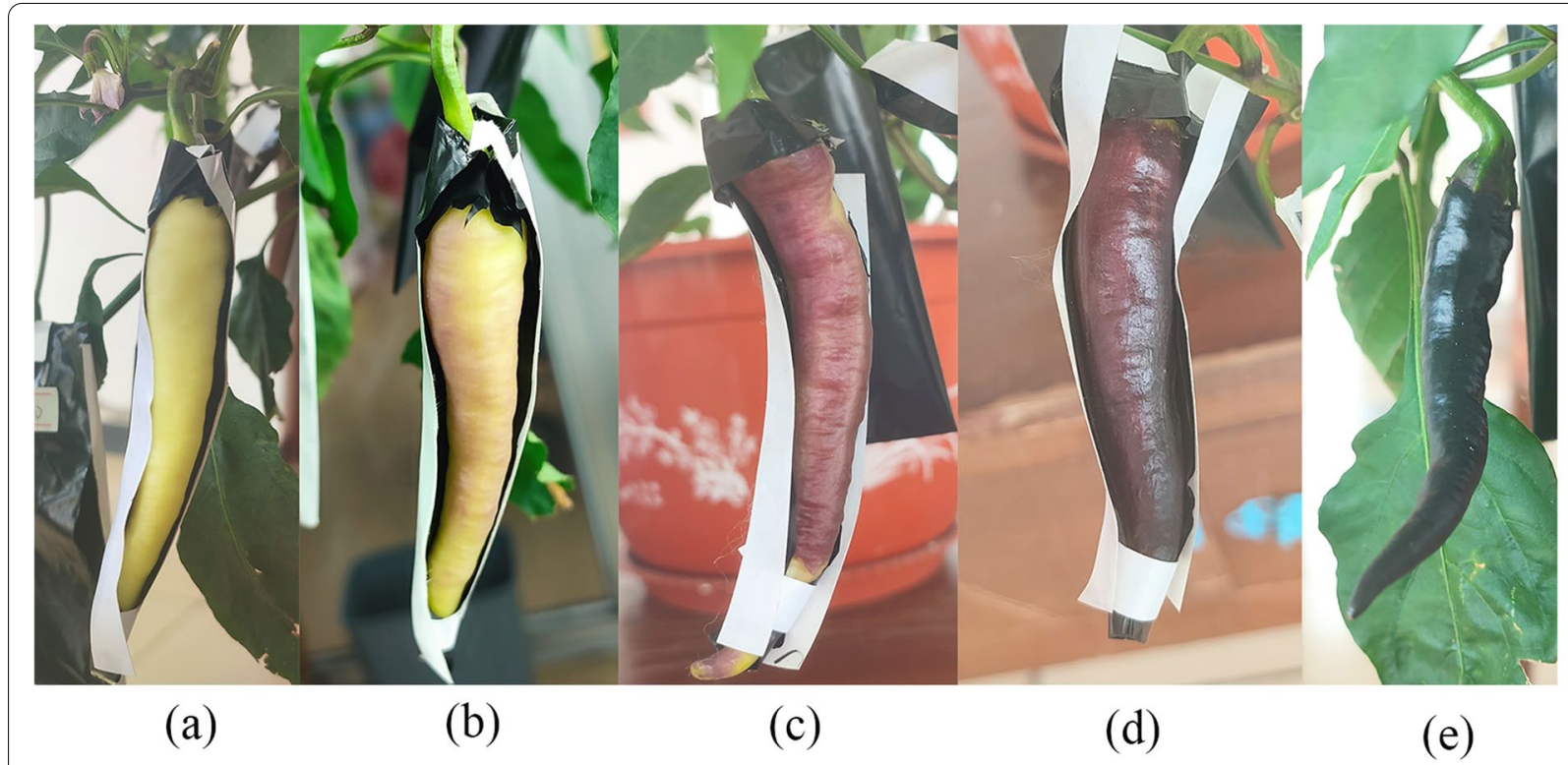

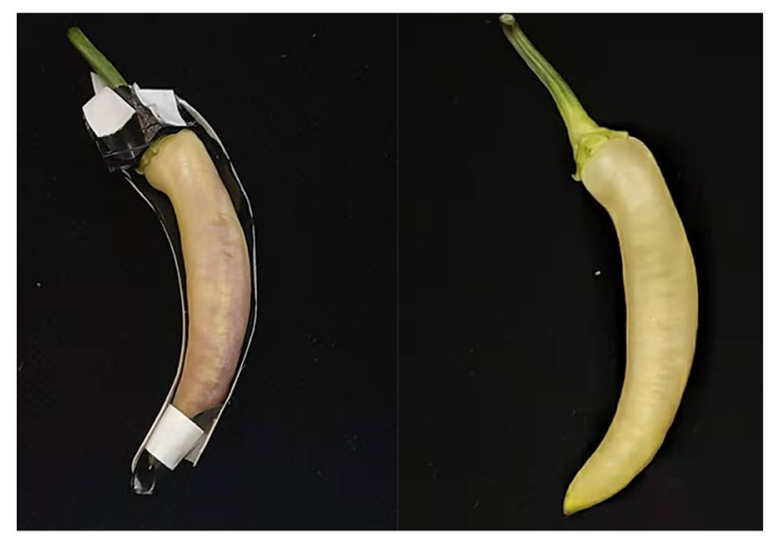

(f)

(g)

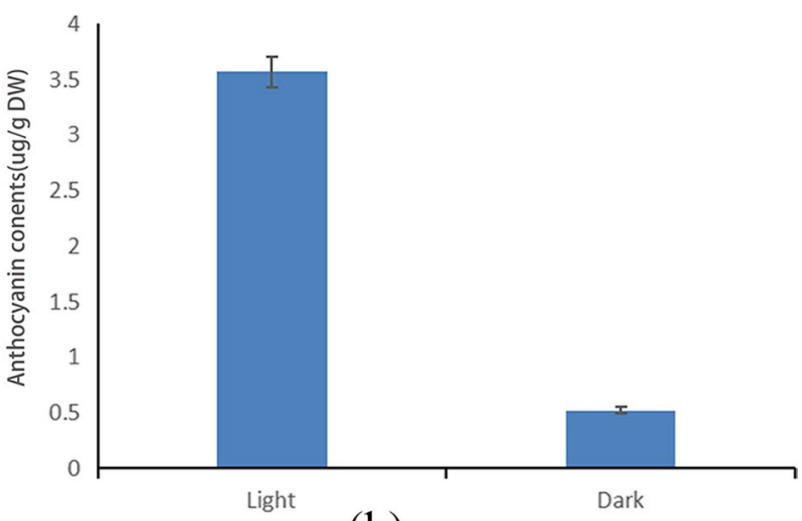

(h)

Fig. 1 Response of the HNUCA7454 to light treatment. a $0 \mathrm{~h}$ of light, yellowish-white fruit. b $48 \mathrm{~h}$ of light, light green, and light purple fruit. c $72 \mathrm{~h}$ of light, purple fruit. $\mathbf{d} 7$ days of light, purple-black fruit. e unbagged fruit. $\mathbf{f} 48 \mathrm{~h}$ of the light side. $\mathbf{g} 48 \mathrm{~h}$ of the dark side. $\mathbf{h}$ Total-Content of Anthocyanins of fruit after $48 \mathrm{~h}$ of light

93.71\%, and the GC percentage was between 41.74 and 42.11\% (Table S1). For miRNA sequencing, the raw reads of these six samples ranged from 1346 to 20.881 million, resulting in RNA-Seq clean bases of 0.673-1.044G. The percentage of Q20 exceeded 98.66\%, the percentage of Q30 exceeded 95.81\%, and the percentage of GC was between 47.58 and $50.46 \%$ (Table S2). For strandspecific RNA sequencing, more than $89.4 \%$ of the clean reads were successfully mapped to the reference genome, of which at least 73.36 and $13.72 \%$ of the clean reads were located uniquely and multiple times, respectively (Table S3). For miRNA sequencing, more than $90.92 \%$ of the reads were successfully mapped to the reference genome, of which at least 67.18 and $22.08 \%$ of the reads were mapped to the strand with the same reference sequence direction and the strand with the opposite direction of the reference sequence respectively (Table S4).

\section{Analysis of DE mRNAs}

From the differentially expressed (DE) mRNAs analysis between D48 and L48, we detected a total of $1341 \mathrm{DE}$ mRNAs (Table S5), of which 1102 were up-regulated and 239 were down-regulated (Fig. 3a). The Venn diagram shows the number of all mRNAs expressed commonly and independently between the two groups. Most genes were expressed simultaneously in D48 and L48 (Fig. 3b). 


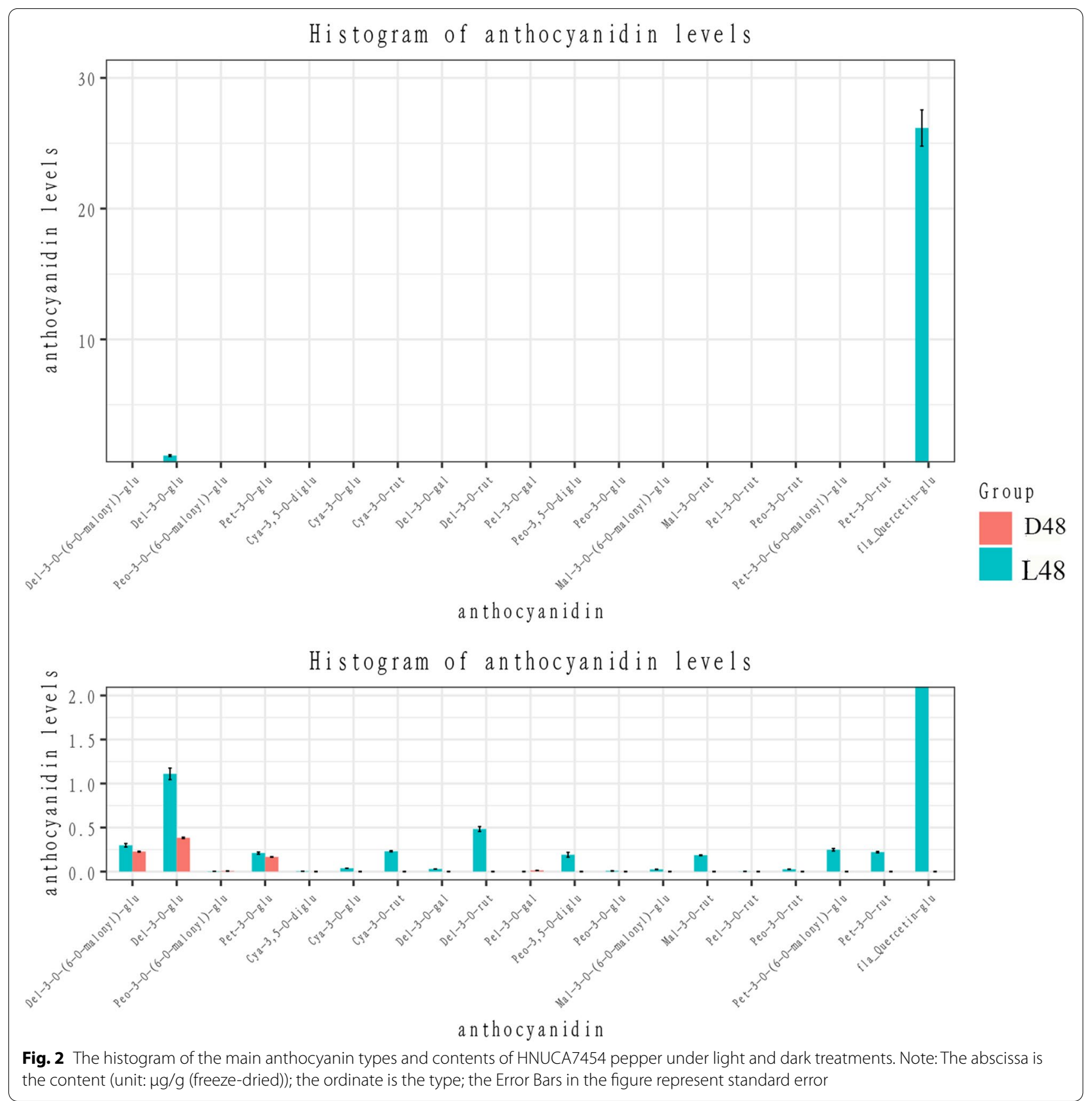

Analysis of GO and KEGG corresponding genes of mRNA with a significant difference under light conditions

From the GO map analysis of the differentially expressed genes (Fig. S1), biological processes that were significantly enriched include single-organism metabolic process (GO:0044710), oxidation-reduction process (GO:0055114), metabolic process (GO:0008152), small molecule metabolic process (GO:0044281), biological process (GO:0008150), nitrogen compound metabolic process (GO:1901564), nonacid metabolic process (GO:0043436), organic acid metabolic process (GO:0006082). reductionist activity (GO:0016491), Co-enzyme binding (GO:0050662), co-factor binding (GO:0048037), and catalytic activity (GO:0003824) are significantly enriched in molecular functions. Significantly enriched in cellular components are Photostatted (GO:0009521), photosynthetic membrane (GO:0034357), thyroidal (GO:0009579), thyroidal part (GO:0044436), Photostatted II (GO:0009523), lactoprotein complex (GO:0030529), thylakoids, and other parts that are 


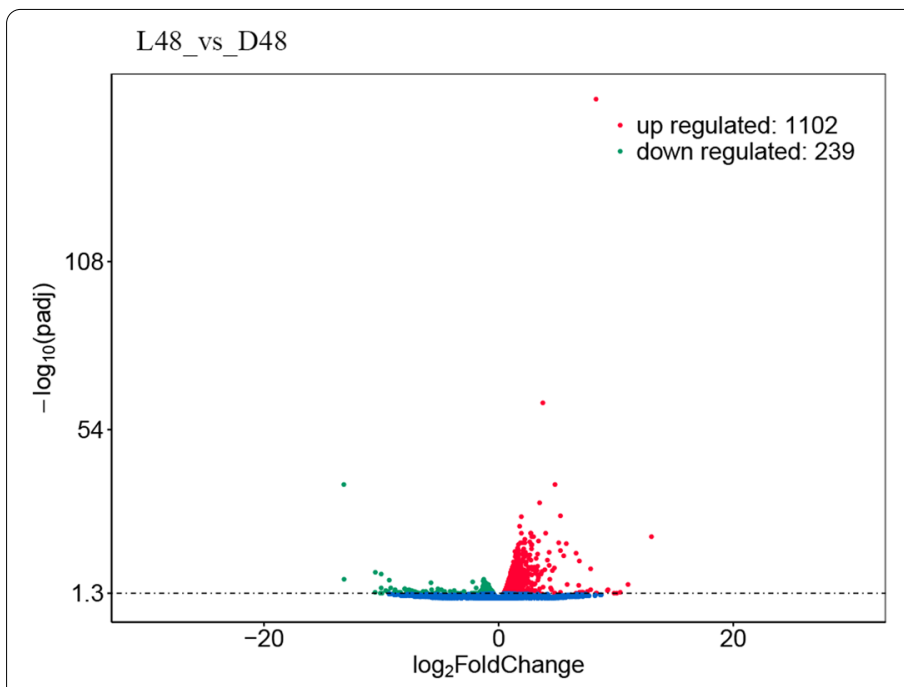

(a)

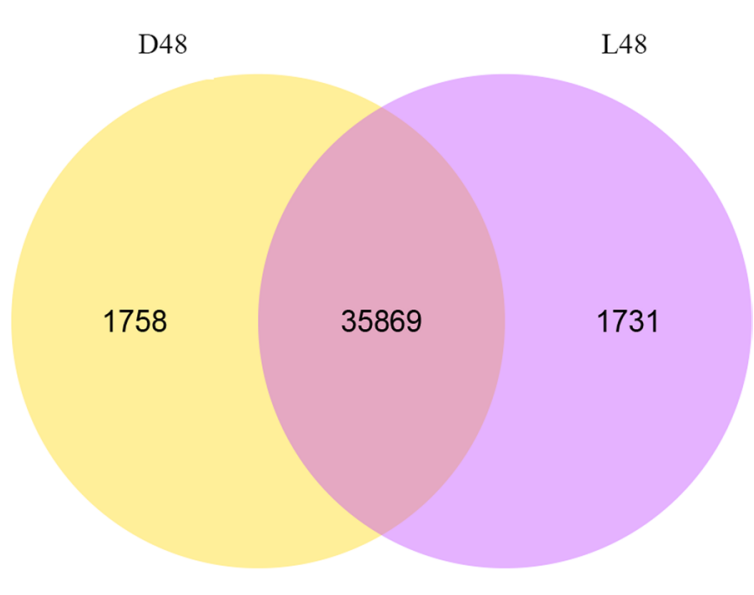

(b)

Fig. 3 D48 and L48 mRNA analysis. a D48 and L48 DE mRNA volcano map. Significantly up-regulated and significantly down-regulated mRNAs are represented by red and green dots, respectively. Blue dots represent mRNAs that are not significantly different. $\mathbf{b}$ Venn diagram of the mRNA expression quantity of all D48 and L48 read count values greater than or equal to 1

significantly enriched in cellular components may also be related to the need to promote the transport of flavonoids through vesicles or transporters [7, 20,21].

Differentially expressed mRNAs are annotated to 98 pathways from the Kyoto Encyclopedia of Genes and Genome pathway (Table S6), of which 10 pathways are significantly enriched. The significant enrichment of differentially expressed genes in the Flavonoid biosynthesis (sly00941) pathway affected pepper fruit color. In this pathway, the genes encoding SHT, shikimate O-hydroxycinnamoyl transferase-like; AT-like, acyl sugar acyltransferase 3-like; CCoAOMT, probable caffeoyl-CoA O-methyltransferase At4g26220 isoform X1; CHI, chalcone--flavanone isomerase isoform $\mathrm{X} 1$; $\mathrm{CHS1B}$, chalcone synthase 1B; CHS2, chalcone synthase 2; CYP98A2-like, cytochrome P450 98A2-like; DFR, dihydroflavonol-4-reductase; F3'5'H, flavonoid 3'\%2C5'-hydroxylase 2; F3H, naringenin\%2C2-oxoglutarate 3-dioxygenase; F3'M, flavonoid 3'-monooxygenase; LDOX, leucoanthocyanidin dioxygenase [22]; TCM, trans-cinnamate 4-monooxygenase; TCM-like, trans-cinnamate 4-monooxygenase-like; were significantly up-regulated. Details of differential genes in the Flavonoid biosynthesis (sly00941) pathway are presented in Table S7.

The early biosynthesis genes (EBGs) [23] of the flavonoid synthesis pathway include $\mathrm{CHS}, \mathrm{CHI}, \mathrm{F} 3 \mathrm{H}$, $F 3^{\prime} 5^{\prime} H$, and Late Biosynthesis Genes (LBGs) [23] including $D F R, L D O X$, were significantly up-regulated under the influence of light (Figs. 4 and 5; Fig. S2). In the pathway diagram of sly00941, we also found two cytochrome P450 enzymes, including F3'M (also known as $C Y P 706 C$ ) and CYP98A2. F3'M is believed to be closely related to the flavonoid $3^{\prime}, 5^{\prime}$-hydroxylase $\left(\mathrm{F}^{\prime}{ }^{\prime}{ }^{\prime} \mathrm{H}\right)[24,25]$, that guides and promotes the synthesis of anthocyanins [26]. In addition, photosynthesis (sly00195; Fig. S3), glyoxylate and dicarboxylate metabolism (sly00630; Fig. S4), carbon fixation in photosynthetic organisms (sly00710; Fig. S5), porphyrin and chlorophyll metabolism (sly00860; Fig. S6), carotenoid biosynthesis (sly00906; Fig. S7), metabolic pathways (sly01100), ubiquinone and another terpenoid-quinone biosynthesis (sly00130), carbon metabolism (sly01200) and biosynthesis of secondary metabolites (sly01110) may also respond to light.

\section{DE mRNAs encoding transcription factors}

Among the DE mRNAs encoding transcription factors obtained by transcriptome sequencing, a total of 402 DE mRNAs were predicted to be related to $53 \mathrm{TF}$ families (Table S8). The top seven transcription factor family types with the highest expression abundance were MYB family with 30 DE mRNAs, C2C2-GATA family with 26 DE mRNAs, $C 3 H$ family with 26 DE mRNAs, $m T E R F$ family with 25 DE mRNAs, $b Z I P$ family with 21 DE mRNAs, Orphans family with 20 DE mRNAs, bHLH family with 19 DE mRNAs, and the WRKY family has 9 DE mRNAs in response to different light conditions. 


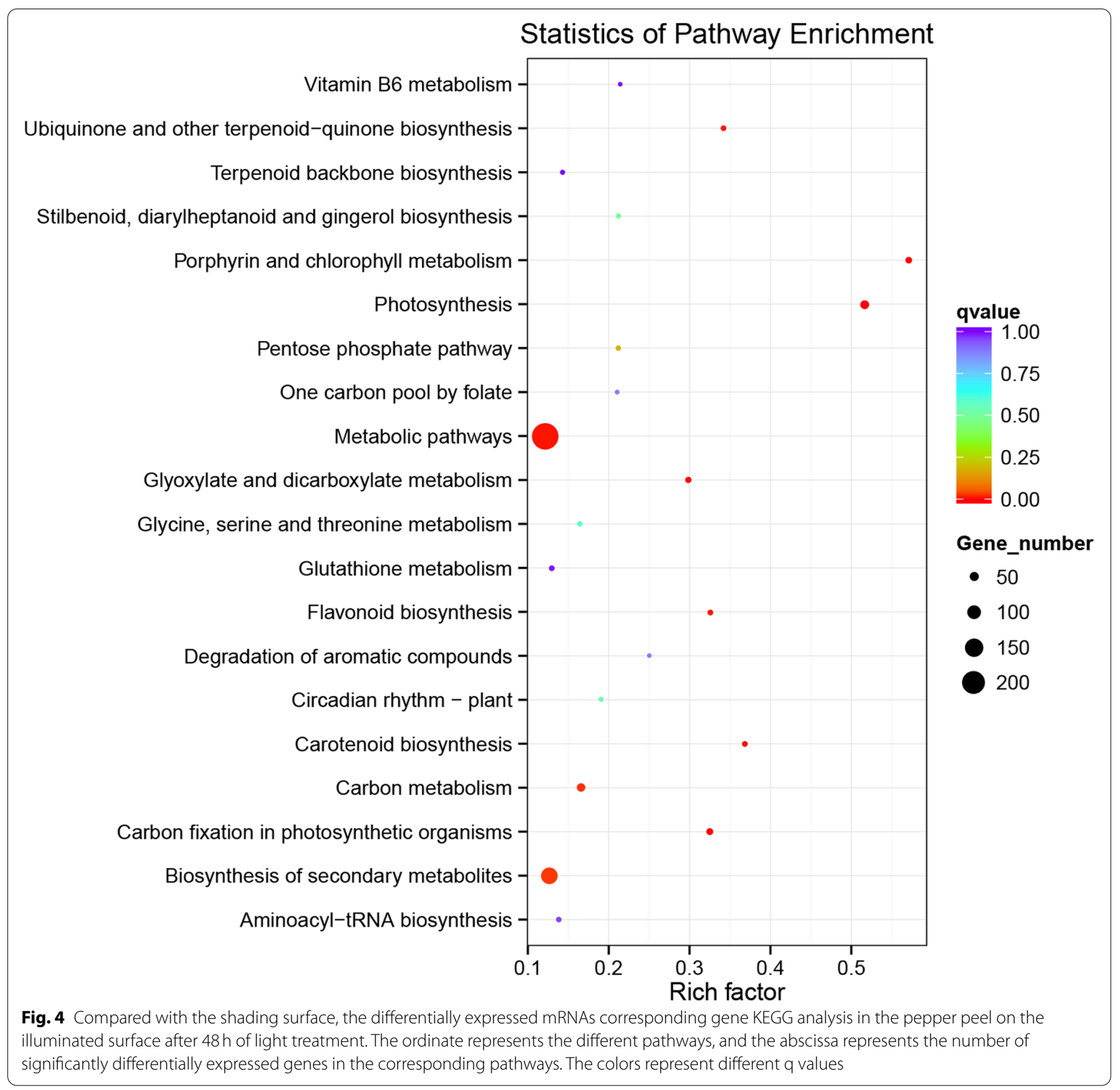

\section{Analysis of DE IncRNAs, DE miRNAs and DE circRNAs}

Figure S8a-d shows the Volcano and Venn plots of DE lncRNAs and DE miRNAs in the light treatment group compared with the shading group, respectively. We identified 172 DE lncRNAs (88 up-regulated and 84 downregulated) between D48 and L48. Figure S8a and b show the Volcano and Venn plots of DE lncRNAs, respectively. The detailed data of lncRNAs with significant differences between D48 and L48 are shown in Table S9.

Table S10 shows all the information of DE miRNAs. In total $28 \mathrm{DE}$ miRNAs including 4 up-regulated and 24 down-regulated were detected. Details of DE circRNAs are shown in Table 1 , with a total of 8 DE circRNAs (1 up-regulated and 7 down-regulated).

We use the co-localization and co-expression of DE IncRNAs and protein-coding genes to predict its biological function. Figure S9c represents the Venn diagram of the cross-analysis of DE lncRNAs, mRNAs and DEmRNAs. There are 259 up-regulated DEmRNAs with up-regulated lncRNAs, 179 down-regulated mRNAs with down-regulated lncRNAs, 43 up-regulated mRNAs with down-regulated lncRNAs, and 5 down-regulated 


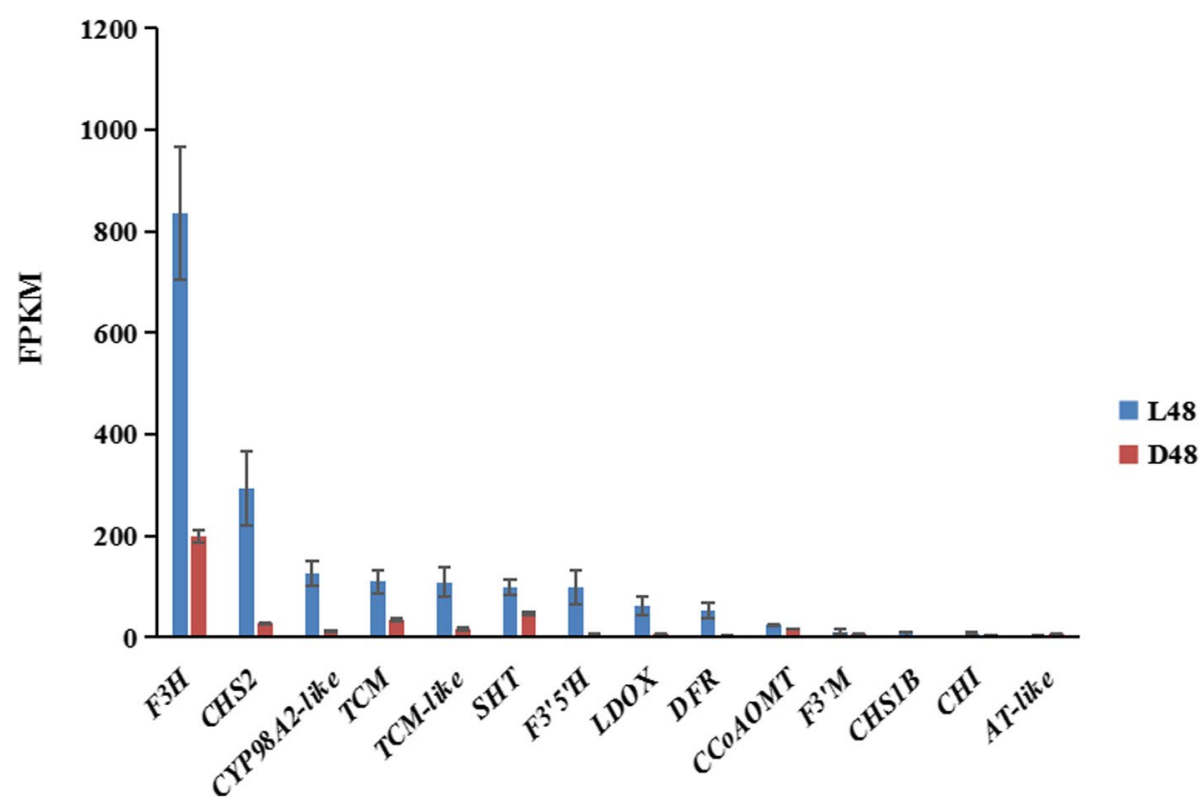

Fig. 5 The FPKM of all genes expressed significantly differently in the sly00941 pathway. SHT (XM_016684802.1), AT-like (XM_016704776.1), CCOAOMT (XM_016698340.1,XM_016698341.1),CHI (XM_016697794.1,XM_016697793.1),CHS2 (XM_016718139.1), CHS1B (XM_016710598.1), CYP98A2-like (XM_016688489.1), DFR (XM_016705224.1), F3'5'H (XM_016693437.1), F3H (XM_016705025.1), F3'M (XM_016707872.1), LDOX (XM_016712446.1), TCM (XM_016722116.1) and TCM-like (XM_016722117.1)

mRNAs with up-regulated lncRNAs (Table S11). Figure S8c shows the Volcano map of DE miRNAs. There are 3 up-regulated DEmRNAs with up-regulated miRNAs, 29 down-regulated mRNAs with down-regulated miRNAs, 130 up-regulated mRNA with down-regulated miRNAs (Table S12).

\section{Function prediction of DE IncRNAs and DE miRNAs}

(Fig. S10)

The GO enrichment analysis of DE lncRNA candidate target genes showed that the mRNA of differentially expressed lncRNAs were mainly enriched in BPs. RNA metabolic process (GO:0016070), gene expression (GO:0010467), cellular nitrogen compound biosynthetic process (GO:0044271), regulation of cellular process (GO:0050794), regulation of biological process (GO:0050789), etc. were enriched.

Through the GO enrichment analysis of DE miRNA candidate target genes, the most significantly enriched MFs are ADP binding (GO:0043531), adenyl nucleotidebinding (GO:0030554), and adenyl ribonucleotide binding (GO:0032559). The most significantly enriched BPs include regulation of cellular process (GO:0050794), biological regulation (GO:0065007), regulation of biological process (GO:0050789), pigment catabolic process (GO:0046149), and chlorophyll catabolic process (GO:0015996).

Table 1 The detailed information of DE CircRNAs between L48 and D48

\begin{tabular}{|c|c|c|c|c|c|c|}
\hline circRNA ID & L48_readcount & D48_readcount & log2FoldChange & pval & padj & Regulation \\
\hline novel_circ_0000040 & 29.00983176 & 0 & 5.2515 & 0.009214 & 0.76789 & up \\
\hline novel_circ_0000092 & 0 & 4.754184168 & -4.5254 & 0.020587 & 0.76789 & down \\
\hline novel_circ_0000320 & 0 & 7.160391679 & -4.931 & 0.010372 & 0.76789 & down \\
\hline novel_circ_0000357 & 0 & 3.760356755 & -4.1972 & 0.035439 & 0.76789 & down \\
\hline novel_circ_0000786 & 0 & 4.053774032 & -4.1257 & 0.040931 & 0.76789 & down \\
\hline novel_circ_0000960 & 0 & 5.761819822 & -4.7911 & 0.012675 & 0.76789 & down \\
\hline novel_circ_0001250 & 0 & 5.821660645 & -4.6149 & 0.018678 & 0.76789 & down \\
\hline novel_circ_0001268 & 0 & 3.678145014 & -4.1616 & 0.037399 & 0.76789 & down \\
\hline
\end{tabular}


We use KEGG enrichment analysis to determine the most important biochemical metabolic pathways and signal transduction pathways related to specific genes. Most mRNAs were significantly enriched in pathways including photosynthesis-antenna proteins (sly00196), porphyrin and chlorophyll metabolism (sly00860), ubiquinone and other terpenoid-quinone biosynthesis (sly00130), spliceosome (sly03040), circuit rhythm-plant (sly04712), photosynthesis (sly00195), flavonoid biosynthesis (sly00941), non-homologous end-joining (sly03450). plant-pathogen interaction (sly04626) and spliceosome (sly03040) pathways were both significant enriched.

Table S13 shows the detailed information of lncRNA and its candidate targeted mRNA in the flavonoid biosynthesis (sly00941) pathway. This pathway includes 25 genes including SHT, AT-like, CCoAOMT, CHI, CHS1B, CHS2, CYP98A2-like, DFR, F3'5'H, F3H, F3'M, FLS [27], LDOX, TCM, TCM-like, etc. may be regulated and expressed by lncRNA. These genes may be the key nodes for lncRNA to regulate anthocyanin synthesis. The plant-pathogen interaction (sly04626) pathway, which is significantly enriched in DE miRNAs, may be related to the stress response of plants in response to strong light. The process of plant defense against pathogens and UV damage may have a common regulatory pathway [28]. A series of biological and other non-biological reactions accompanying the synthesis of anthocyanins may make plants more resistant to the damage caused by multiple threats.

\section{Association network analysis}

Long non-coding RNA acts as a competitive endogenous RNA to regulate gene expression. To predict interaction between miRNA and mRNA, lncRNA and miRNA. We constructed three ncRNA-miRNA-mRNA gene interaction networks which may be related to light response. Involving 28 differentially expressed mRNAs, 6 differentially expressed lncRNAs, and 4 differentially expressed miRNAs. Three regulatory networks with sly-miR5303, stu-miR5303g, stu-miR7997a, and stu-miR7997c as the core were constructed (Fig. 6).

\section{qRT-PCR analysis of genes related to anthocyanin metabolism in pepper}

For qRT-PCR verification a total of 12 genes associated with synthetic and regulatory pathway of anthocyanin metabolism were selected. The findings revealed that all 12 DEGs expression data of qRT-PCR complied with the RNA-seq expression data (Fig. S11).

\section{Discussion}

The enrichment of anthocyanins on the surface of plants can reduce the damage caused by strong light to plant cells and protect plant growth [4]. The isotomycin rich in purple pepper has a certain effect on reducing inflammation, promoting antioxidant enzyme activity, and preventing obesity [29]. Anthocyanins are beneficial to the health of animals and humans [30] and are considered

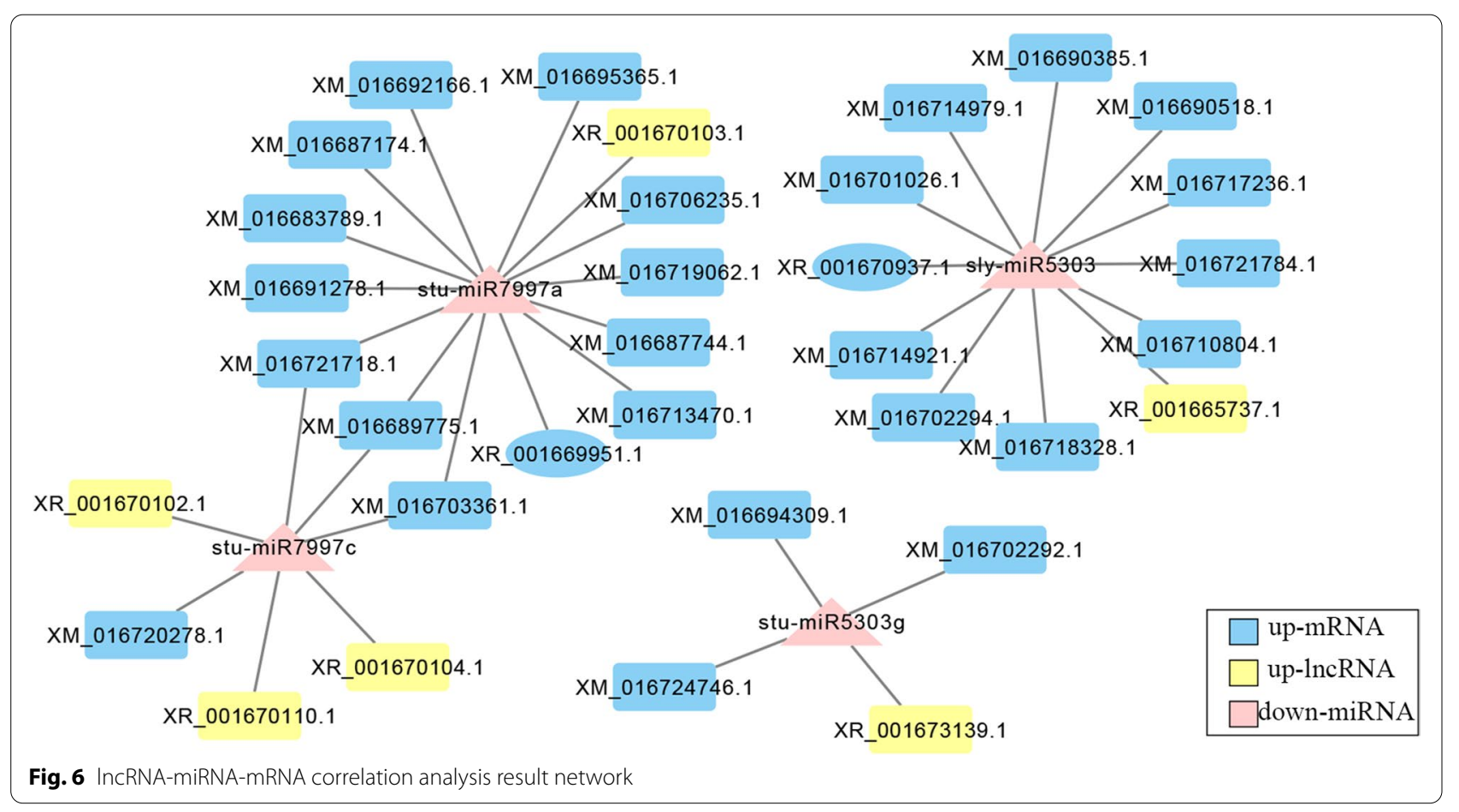


green and healthy plant nutrients [31]. Anthocyanins are also natural food colorants and are safer for the human body.

Controlling the synthesis of anthocyanins has always been an issue of great concern. This study analyzed the anthocyanin formation under different light treatments to elaborate the molecular mechanism of light-dependent anthocyanin formation in pepper fruits. Two weeks after bagging, HNUCA7454 capsicum fruits colored quickly and anthocyanins synthesized within $48 \mathrm{~h}$ after receiving light. We determined the types and contents of synthetic anthocyanins on the light side and the dark side of fruit. At the same time, anthocyanin determination and transcriptome analysis were performed on the fruits under the different light treatment conditions. Eighteen types of flavonoids are synthesized on the light exposed surface. In the light exposed surface isoquercetin was identified as major accumulated anthocyanin. Whereas pelargonidin 3-O-galactoside synthesized only in the shaded surface. Furthermore, 1341 DE mRNAs, some lncRNAs and miRNAs were also identified significantly altered under different light conditions.

\section{The role of IncRNA and miRNA in anthocyanin synthesis and transport and other metabolic pathways}

At present, the basic pathways of anthocyanin biosynthesis are relatively comprehensive, but there are few studies on anthocyanin synthesis and transportation and the mutual influence on other metabolic pathways. Noncoding RNAs (ncRNAs), can also play a vital role in regulating gene expression [32]. Recent studies have shown that miRNAs are involved in anthocyanin biosynthesis. In $A$. thaliana, increased miR156 activity promotes the accumulation of anthocyanins, whereas reduced miR156 activity directs synthesis toward flavonols [33]. The miR156 target, SQUAMOSA PROMOTER-BINDING PROTEIN-LIKE 9 (SPL9), has also been shown to suppress anthocyanin accumulation by preventing the expression of anthocyanin biosynthetic genes through destabilization of a MYB-bHLHWD40 transcriptional activation complex [33]. In our study, we identified 6 differentially expressed lncRNAs, and 4 differentially expressed miRNAs. Among the 28 mRNAs in the 'IncRNA-miRNA-mRNA' interaction network, some genes are related to pathways such as Protein export, Endocytosis, Ubiquitin mediated proteolysis, Plant-pathogen interaction and Plant hormone signal transduction. Studies have shown that the accumulation of anthocyanins is related to the metabolism of plant hormones such as jasmonic acid [34, 35], abscisic acid [36], and ethylene [37], strong light [38, 39], mechanical damage, and pathogens [40].
However, the role of lncRNA in light regulation of anthocyanin biosynthesis and transport still need further verification.

\section{TFs response to light}

Transcription factors are the key to the biosynthesis of flavonoids. The MBWs $[14,16,19,31]$ ternary complex composed of $M Y B, b H L H$, and WDR has the greatest impact on the synthesis of flavonoids. It has been reported that a variety of MYBs can significantly regulate the synthesis of flavonoids [41-43]. R2R3MYB protein promotes the accumulation of anthocyanin products in the flavonoid synthesis process of various crops such as pepper, radish [9], and tomato [44], while the effect of R3-MYB is just the opposite. The transcription factors AtMYB11, AtMYB111, and AtMYB12 derived from Arabidopsis can promote the accumulation of flavonoids in tobacco [45] and delay the metabolism of narcissus flavonoids by inhibiting the biosynthesis of flavonols [29]. We identified 30 significantly different $M Y B$ transcription factors, including MYB4-like (XM_016725242.1), MYB113-like (XM_016689220.1), and MYB308-like (XM_016696983.1, XM_016702244.1). Which are the most abundant among all transcription factors. Existing studies have shown that MYB4 [46] participates in the balanced regulation of the accumulation of anthocyanins and proanthocyanidins in mulberry fruits [47]. Another study has shown that MYB4 plays a bidirectional role in regulating the synthesis of Arabidopsis flavonoids [48]. In our experimental results, MYB4 was significantly down-regulated, showing its negative regulatory function. MYB113 plays a positive role in the process of UV-B-induced Arabidopsis anthocyanin synthesis [49] and regulates anthocyanin synthesis [50].

MYB308 is related to the biosynthesis of flavonoids [51, 52]. We found that MYB308 has both significantly up-regulated and down-regulated genes in the process of flavonoid synthesis, indicating that it may have the same bidirectional function of regulating flavonoid synthesis as MYB4.

In addition, among the $19 \mathrm{bHLH}$ transcription factors that we identified with significant differences, most importantly EGL1 (XM_016711673.1) expression correlated with degree of anthocyanin synthesis which indicates its important role in the light regulated flavonoid biosynthesis pathway.

\section{Conclusions}

Possible light-regulated flavonoid biosynthesis earlystage response genes, including structural genes, transcription factor families, lncRNA, miRNA, and circRNA 
were identified through strand specific transcriptome analysis. We determined that the main anthocyanin involved in the visible light response of HNUCA7454 pepper is delphinidin 3-O-glucosideIn this study, we identified delphinidin 3-O-glucoside as the major light regulated anthocyanin in pepper fruits. Furthermore, SHT, AT-like, CCoAOMT, CHI, CHS1B, CHS2, CYP98A2-like, DFR, F3'5' $H$, F3H, F3'M, and FLS were identified as major players in light-regulated anthocyanin biosynthesis. Moreover, the transcription factors including MYB4-like, MYB113-like, MYB308-like, and $E G L 1$ were probably involved in the regulation of flavonoid metabolism. Overall results from this study promote understanding of photo-regulated molecular control of anthocyanin biosynthesis and provide valuable knowledge for in depth understanding of anthocyanin molecular regulation.

\section{Materials and methods Plant materials}

The pepper germplasm HNUCA7454 (Capsicum annuum) was collected from the germplasm bank of Hainan University, Hainan Province $\left(20^{\circ} 3^{\prime} 38^{\prime \prime}\right.$ north latitude, $110^{\circ} 19^{\prime} 8^{\prime \prime}$ east longitude). The HNUCA7454 entered the flowering stage about 80 days after sowing. Thirty flowers open on the same day were pollinated and marked simultaneously. Three days later, the pollinated flowers were bagged with a black plastic bag. After 14 days of bagging, half of the black plastic bags on the fruits were removed (Fig. 1). The fruits were treated with light for $48 \mathrm{~h}$ and the controlled fruits were collected and stored at $-80^{\circ} \mathrm{C}$ for subsequent metabolite extraction, RNA isolation, and RNA-Seq analysis. The samples on the illuminated side and shading side were named L48 and D48, respectively. Three biological replicates were used to carry out further analysis.

\section{Determination of total anthocyanin}

The total anthocyanin content was measured by a method previously described [53]. After grinding $0.2 \mathrm{~g}$ of the sample, it was added to $1.5 \mathrm{ml}$ of a solution containing methanol: acetic acid (99:1, v/v), and then placed at $4{ }^{\circ} \mathrm{C}$ for overnight extraction. The extract was filtered, and then the absorbance of the extract was measured at 530,620 , and $650 \mathrm{~nm}$ using a DU 800 spectrophotometer (Beckman Coulter, Fullerton, California). Total anthocyanin $=[($ OD $530-$ OD 650) $-0.2 \times$ OD $650-$ OD 620).

\section{Determination of flavonoids by LC-ESI-MS/MS}

The flavonoids in the collected samples were analyzed by Met Ware (Wuhan, China). Ultra performance liquid chromatography (UPLC) (ExionLC ${ }^{\mathrm{TM}} \mathrm{AD}$, https:// sciex.com.cn/) and tandem mass spectrometry (MS/MS)
(QTRAP ${ }^{\circledR} 6500+$, HTTP://sciex.com.cn/) (LC-ESI-MS/ MS) was used for relative quantification of flavonoids [54]. The sample was freeze-dried in a vacuum and then ground and weighed out $50 \mathrm{mg}$ of powder. The sample was extracted in a $500 \mu \mathrm{l}$ extraction solution (50\% methanol aqueous solution containing $0.1 \%$ hydrochloric acid) and then centrifuged to extract the supernatant. Finally, the supernatant was filtered and stored with a microporous membrane (pore size $0.22 \mu \mathrm{m}$ ) for LC-MS/MS analysis.

The liquid phase conditions mainly include: LC: ACQUITY BEH C18 $(1.7 \mu \mathrm{m}, 2.1 \mathrm{~mm} * 100 \mathrm{~mm})$; mobile phase: phase $\mathrm{A}$ is ultrapure water (adding $0.1 \%$ formic acid), phase $B$ is methanol (adding $0.1 \%$ formic acid); Degradation: B phase ratio is $0.00 \mathrm{~min}, 5 \%, 6.00 \mathrm{~min}$ increased to $50 \%, 12.00 \mathrm{~min}$ increased to $95 \%$, maintained for $2 \mathrm{~min}$, decreased 14 to $5 \%$, equilibrated for $2 \mathrm{~min}$; flow rate $0.35 \mathrm{ml} / \mathrm{min}$; column temperature is $40^{\circ} \mathrm{C}$. The injection volume is $2 \mu \mathrm{l}$.

Mass spectrometry conditions mainly include Electrospray Ionization (ESI) temperature $550^{\circ} \mathrm{C}$, mass spectrometry voltage $5500 \mathrm{~V}$ in positive ion mode, and Curtain Gas (CUR) 35 psi. In Q-Trap 6500+, each ion pair is scanned based on the optimized declustering voltage (Declustering Potential, DP) and collision energy (Collision Energy, CE).

\section{RNA isolation and library preparation}

The samples were analyzed and processed by Novogene Bioinformatics Technology Co. Ltd. (Beijing, China). The total RNA in the sample was extracted with Trizol Reagent (Invitrogen, Carlsbad, CA, USA), and then evaluated by agarose gel electrophoresis and RNA Nano 6000 detection kit (Agilent Technologies, CA, USA) [55]. The Illumina Hiseq platform (Illumina, USA) was used to generate a sample library, and the samples had three biological replicates. Sequencing libraries were generated using NEBNext ${ }^{\circledR}$ Multiplex Small RNA Library Prep Set for Illumina ${ }^{\circledR}$ (NEB, USA.). NEB $3^{\prime}$ SR Adaptor was directly and specifically ligated to $3^{\prime}$ end of miRNA, siRNA, and piRNA. Connect the 5 'end adapter to the 5 ' end of miRNA, siRNA and piRNA. The first cDNA strand was then synthesized using M-MuLV Reverse Transcriptase (RNase $\mathrm{H}^{-}$). After PCR amplification DNA fragments corresponding to $140 \sim 160 \mathrm{bp}$ were recovered, library quality was assessed on the Agilent Bioanalyzer 2100 system [56]. The reference genome was downloaded from (https://www.ncbi.nlm.nih.gov/genome/10896).

\section{Differential expression analysis}

DE mRNA and DE lncRNA were measured with the aid of Ballgown. DESeq2 is used to identify DE miRNA and 
DE circRNA. Genes with a $P$-value $<0.05$ are considered to be differentially expressed. Perform ontology (GO) annotation on the GO SEQ R software package, classify genes according to biological processes (BP), cell components (CC), and molecular functions (MF), and predict their functions. KEGG (Kyoto Encyclopedia of Genes and Genomes) is the main public database related to this pathway (https://www.genome.jp/kegg/; 20-04-2018). Kobas v2.0 software provides help for the enrichment analysis of DE mRNA corresponding genes and DElncRNA targeted genes detected in the KEGG pathway. The transcription factor prediction in this experiment was realized by iTALK 1.2 software.

\section{Construction of competitive endogenous RNA regulatory network}

According to the hypothesis of competitive endogenous RNA (ceRNA), IncRNA containing one or more miRNA response elements (MRE) in common can compete with mRNA to bind to miRNA to regulate expression. Cytoscape 3.7.1 software was used to construct the regulatory network between lncRNA-miRNA-mRNA.

RNA extraction and quantitative reverse transcription-PCR RNA prep Pure Plant Plus Kit (Tian Gen, Beijing, China) was used to extract total RNA from pepper peel samples. The integrity and purity of RNA were determined by agarose gel electrophoresis and NANODROP 2000 spectrophotometer (Thermo Fisher Scientific, MA, USA), and then quantified before use. The RNA samples were reverse-transcribed to synthesize cDNA by Fast King gDNA Dispelling RT SuperMix (Tian Gen, Beijing, China). Quantitative real-time PCR was performed using the Light Cycler 96 Real-Time PCR System (Roche, Switzerland) instrument. Evaluate the relative expression level of the target gene based on the $2-\Delta \Delta \mathrm{Ct}$ method. Ubi-3 was selected as the internal reference gene to balance the differences between different samples. The primer information for qRT-PCR is given in Table S14.

\footnotetext{
Abbreviations

DEGs: Differentially expressed genes; RNA-seq: RNA sequencing technology; IncRNA: Long non-coding RNA; GO: Gene Ontology; KEGG: Kyoto Encyclopedia of Genes and Genome; ceRNA: Competing endogenous RNA; TFs: Transcription Factors; AT: Acyl sugar acyltransferase 3-like; CCOAOMT: Probable caffeoyl-CoA O-methyltransferase At4g26220 isoform X1; CHI: Chalcone--flavanone isomerase isoform X1; CHS1B: Chalcone synthase 1B; CYP98A2-like: Cytochrome P450 98A2-like; DFR: Dihydroflavonol-4-reductase; F3'5'H: Flavonoid 3'\%2C5'-hydroxylase 2; F3H: Naringenin\%2C2-oxoglutarate 3-dioxygenase; F3'M: Flavonoid 3'-monooxygenase; LDOX: Leucoanthocyanidin dioxygenase; TCM: Trans-cinnamate 4-monooxygenase.
}

\section{Supplementary Information}

The online version contains supplementary material available at https://doi. org/10.1186/s12870-021-03423-6.

Additional file 1: Table S1. Quality evaluation of sample sequencing output data for strand-specific RNA libraries.

Additional file 2: Table S2. Quality evaluation of sample sequencing output data for small RNA libraries.

Additional file 3: Table S3. Reads of strand-specific RNA libraries and reference genome comparison list.

Additional file 4: Table S4. Reads of small RNA libraries and reference genome comparison list.

Additional file 5: Table S5. The detailed information of DE mRNAs.

Additional file 6: Table S6. KEGG analysis results of DE mRNA corresponding genes.

Additional file 7: Table S7. Detailed information of differential genes in the Flavonoid biosynthesis pathway.

Additional file 8: Table S8. DE mRNAs encoding transcription factor. Additional file 9: Table S9. The detailed information of DE IncRNAs betweenL48 and D48.

Additional file 10: Table S10. The detailed information of DE miRNAs between $L 48$ and D48.

Additional file 11: Table S11. The detailed information between DE IncRNA and its targeted mRNA.

Additional file 12: Table S12. The information between DE miRNA and its DE targeted mRNA (up-regulation).

Additional file 13: Table S13. The detailed information of IncRNA and its targeted mRNA in the Flavonoid biosynthesis (sly00941) pathway.

Additional file 14: Table S14. The primers information for qRT-PCR of genes related to anthocyanin synthesis.

Additional file 15: Figure S1. Compared with the shading surface, the DE mRNAs in the pepper peel on the shining surface after $48 \mathrm{~h}$ of light treatment corresponds to the GO classification of the gene. The abscissa is the name of the functional classification of gene enrichment. Followed by biological processes (BPs), cellular components (CCs), and molecular functions (MFs). The ordinate is the number and proportion of genes enriched in this function.

Additional file 16: Figure S2. Compared with the light-shielded surface, the KEGG analysis of the differentially expressed mRNAs corresponding genes in the pepper peel on the light-treated surface enriched the "Flavonoid biosynthesis" pathway. KO nodes in the pathway whose expression levels are up-regulated are marked with a red box, and those with up-regulated and down-regulated genes are marked with a yellow box. AT, acyl sugar acyltransferase 3-like; CCOAOMT, probable caffeoyl-CoA O-methyltransferase At4g26220 isoform X1; CHI, chalcone--flavanone isomerase isoform X1; CHS1B, chalcone synthase 1B; CYP98A2-like, cytochrome P450 98A2-like; DFR, dihydroflavonol-4-reductase; F3'5' H, flavonoid 3'\%2C ' -hydroxylase 2; F3H, naringenin $\% 2 \mathrm{C} 2$-oxoglutarate 3-dioxygenase; F3'M, flavonoid 3'-monooxygenase; LDOX, leucoanthocyanidin dioxygenase; TCM, trans-cinnamate 4-monooxygenase

Additional file 17: Figure S3. The photosynthesis pathway enriched by KEGG analysis of DE mRNA corresponding genes.

Additional file 18: Figure S4. The glyoxylate and dicarboxylate metabolism pathway enriched by KEGG analysis of DE mRNA corresponding genes.

Additional file 19: Figure S5. The carbon fixation in photosynthetic organisms pathway enriched by KEGG analysis of DE mRNA corresponding genes.

Additional file 20: Figure S6. The porphyrin and chlorophyll metabolism pathway enriched by KEGG analysis of DE mRNA corresponding genes. 
Additional file 21: Figure S7. The carotenoid biosynthesis pathway enriched by KEGG analysis of DE mRNA corresponding genes.

Additional file 22: Figure S8. D48 and L48 IncRNA and miRNA analysis. a Volcano map of IncRNAs of D48 and L48. Significantly differentially expressed IncRNAs are represented by red dots (up-regulated) and green dots (down-regulated), while non-differentially expressed IncRNAs are represented by blue dots. $\mathbf{b}$ Venn graph of the number of IncRNAs with reading count values greater than or equal to 1 for D48 and L48. c Volcano map of miRNAs of D48 and L48. Significantly differentially expressed miRNAs are represented by red dots (up-regulated) and green dots (downregulated), while non-differentially expressed genes are represented by blue dots. $\mathbf{d}$ Venn map of the number of miRNAs with reading count value greater than or equal to 1 for D48 and L48.

Additional file 23: Figure S9. CircRNAs, DE IncRNA targeted mRNA, DE miRNA targeted mRNA analysis of D48 and L48. a Volcano plot of circRNAs of D48 and L48. Significantly differentially expressed circRNAs are represented by red dots (up-regulated) and green dots (down-regulated), while non-differentially expressed are represented by blue dots. $\mathbf{b}$ Venn map of all circRNAs with reading count values greater than or equal to 1 for D48 and L48. c Venn diagram of the intersection analysis between DE IncRNA targeted mRNA and DE mRNA. $\mathbf{d}$ Venn diagram of the intersection analysis between DE miRNA targeted mRNA and DE mRNA.

Additional file 24: Figure S10. $\mathbf{a}$ and $\mathbf{b} G O$ enrichment of DE IncRNAs and miRNAs on the illuminated surface of HNUCA7454 pepper after $48 \mathrm{~h}$ of light treatment. The ordinate is the rich GO item, and the abscissa is the number of differentially expressed genes in the item and its proportion. Different colors are used to distinguish biological processes and molecular functions. $\mathbf{c}$ and $\mathbf{d}$ are the scatter plots of KEGG enrichment of IncRNAs and miRNAs on the illuminated surface of HNUCA7454 pepper. The vertical axis represents the pathname, and the horizontal axis represents the richness factor. The size of the dot indicates the number of differentially expressed genes in the pathway, and the color of the dot corresponds to the range of different q values.

Additional file 25: Figure S11. qRT-PCR analysis of genes related to anthocyanin metabolism of L48 and D48. a Relative expression analysis of synthetic genes. SHT (XM_016684802.1), AT-like (XM_016704776.1), CHSIB (XM_016710598.1), CYP98A2-like (XM_016688489.1), DFR (XM_016705224.1), F3'5'H (XM_016693437.1), F3H (XM_016705025.1), F3'M (XM_016707872.1), LDOX (XM_016712446.1). b Relative expression analysis of regulatory genes. MYB113-like (XM_016689220.1), MYB308like-1 (XM_016696983.1), MYB308like-2 (XM_016702244.1), The x-axis represents the different treatments, the $y$-axis represents the relative gene expression level and RNA-seq FPKM. Error bars indicate the standard error of the mean $(n=3)$.

\section{Acknowledgements}

Great thanks to anonymous reviewers for their valuable comments.

\section{Authors' contributions}

For research articles: Conceptualization, Z.W., and Y.Z; methodology, Z.W., and Y.Z.; software, Zhang Y.H., H.S., Y.H., Z.Y., S.C, J.Z.; validation, Y.Z., G.Z, M.A.M., W.B. The author(s) read and approved the final manuscript.

\section{Funding}

This work was supported by a grant from the National Key Research and Development Program of China (2018YFD1000800 to Z.W.) and the National Natural Science Foundation of China (31960084 to Z.W.).

\section{Availability of data and materials}

All the original read data have been uploaded to the NCBI serial read file (SRA, https://submit.ncbi.nlm.nih.gov/subs/sra/; this SRA submission will be published on or on 28-05-2025 Issued at the time). The accession numbers are SRR14710538 (L15QN1), SRR14710543 (D15QN1), SRR14710537 (L15QN2), SRR14710536 (L15QN3), SRR14710539 (D15QN3), SRR14710542 (D15QN2), SRR14710532 (L15QN1_raw), SRR14710533 (D15QN3_raw), SRR14710540 (L15QN3_raw), SRR14710535 (D15QN1_raw), SRR14710541 (L15QN2_raw),
SRR14710534 (D15QN2_raw). Other results mentioned in this paper are shown in the article and supplementary figures and tables.

\section{Declarations}

\section{Ethics approval and consent to participate}

Not applicable.

\section{Consent for publication}

This research is not applicable to consent for publication.

\section{Competing interests}

The authors declare no conflict of interest.

\section{Author details}

${ }^{1}$ Key Laboratory for Quality Regulation of Tropical Horticultural Crops of Hainan Province/Engineering Research Center of the Ministry of Education for New Variety Breeding of Tropical Crop, School of Horticulture, Hainan University, Haikou 570228, China. ${ }^{2}$ Institute of Tropical Horticulture Research in Hainan Academy of Agricultural Sciences, Haikou 571100, China.

\section{Received: 1 Auqust 2021 Accepted: 30 December 2021}

Published online: 22 February 2022

\section{References}

1. Tohge T, de Souza LP, Fernie AR. Current understanding of the pathways of flavonoid biosynthesis in model and crop plants. J Exp Bot. 2017;68(15):4013-28.

2. Agati G, Azzarello E, Pollastri S, Tattini M. Flavonoids as antioxidants in plants: Iocation and functional significance. Plant Sci. 2012;196:67-76.

3. Nakabayashi R, Yonekura-Sakakibara K, Urano K, Suzuki M, Yamada Y, Nishizawa T, et al. Enhancement of oxidative and drought tolerance in Arabidopsis by overaccumulation of antioxidant flavonoids. Plant J. 2014;77(3):367-79.

4. Emiliani J, Grotewold E, Falcone Ferreyra ML, Casati P. Flavonols protect Arabidopsis plants against UV-B deleterious effects. Mol Plant. 2013;6(4):1376-9.

5. Wang F, Xu Z, Fan X, Zhou Q, Cao J, Ji G, et al. Transcriptome analysis reveals complex molecular mechanisms underlying UV tolerance of wheat ( Triticum aestivum, L.). J Agric Food Chem. 2019;67(2):563-77.

6. Liu H, Du Y, Chu H, Shih CH, Wong YW, Wang M, et al. Molecular dissection of the pathogen-inducible 3-deoxyanthocyanidin biosynthesis pathway in sorghum. Plant Cell Physiol. 2010;51(7):1173-85.

7. Ku YS, Ng MS, Cheng SS, Lo AW, Xiao Z, Shin TS, et al. Understanding the composition, biosynthesis, accumulation and transport of flavonoids in crops for the promotion of crops as healthy sources of flavonoids for human consumption. Nutrients. 2020;12(6):1717.

8. Appelhagen I, Wulff-Vester AK, Wendell M, Hvoslef-Eide AK, Russell J, Oertel A, et al. Colour bio-factories: towards scale-up production of anthocyanins in plant cell cultures. Metab Eng. 2018;48:218-32.

9. Gomes JVP, Rigolon TCB, Souza M, Alvarez-Leite Jl, Lucia CMD, Martino $H S D$, et al. Antiobesity effects of anthocyanins on mitochondrial biogenesis, inflammation, and oxidative stress: a systematic review. Nutrition. 2019;66:192-202.

10. Tang B, Li L, Hu Z, Chen Y, Tan T, Jia Y, et al. Anthocyanin accumulation and transcriptional regulation of anthocyanin biosynthesis in purple pepper. J Agric Food Chem. 2020;68(43):12152-63.

11. Falcone Ferreyra ML, Rius SP, Casati P. Flavonoids: biosynthesis, biological functions, and biotechnological applications. Front Plant Sci. 2012;3:222

12. Alseekh S, Perez de Souza L, Benina M, Fernie AR. The style and substance of plant flavonoid decoration; towards defining both structure and function. Phytochemistry. 2020;174:112347.

13. Shi MZ, Xie DY. Biosynthesis and metabolic engineering of anthocyanins in Arabidopsis thaliana. Recent Pat Biotechnol. 2014;8(1):47-60.

14. Xu W, Grain D, Bobet S, Le Gourrierec J, Thevenin J, Kelemen Z, et al. Complexity and robustness of the flavonoid transcriptional regulatory 
network revealed by comprehensive analyses of MYB-bHLH-WDR complexes and their targets in Arabidopsis seed. New Phytol. 2014;202(1):132-44.

15. Liu Y, Hou H, Jiang $X$, Wang P, Dai $X$, Chen W, et al. A WD40 repeat protein from camellia sinensis regulates anthocyanin and proanthocyanidin accumulation through the formation of MYB(-)bHLH(-)WD40 ternary complexes. Int J Mol Sci. 2018;19(6):1686.

16. Ichino T, Fuji K, Ueda H, Takahashi H, Koumoto Y, Takagi J, et al. GFS9/TT9 contributes to intracellular membrane trafficking and flavonoid accumulation in Arabidopsis thaliana. Plant J. 2014;80(3):410-23.

17. Feng R, Zhang C, Ma R, Cai Z, Lin Y, Yu M. Identification and characterization of WD40 superfamily genes in peach. Gene. 2019;710:291-306.

18. Fu M, Yang $X$, Zheng J, Wang L, Yang $X$, Tu Y, et al. Unraveling the regulatory mechanism of color diversity in camellia japonica petals by integrative transcriptome and metabolome analysis. Front Plant Sci. 2021;12:685136

19. Xu W, Dubos C, Lepiniec L. Transcriptional control of flavonoid biosynthesis by MYB-bHLH-WDR complexes. Trends Plant Sci. 2015;20(3):176-85.

20. Zhao J, Dixon RA. MATE transporters facilitate vacuolar uptake of epicatechin 3'-O-glucoside for proanthocyanidin biosynthesis in Medicago truncatula and Arabidopsis. Plant Cell. 2009;21(8):2323-40.

21. Wei Y, Ang EL, Zhao H. Recent developments in the application of P450 based biocatalysts. Curr Opin Chem Biol. 2018;43:1-7.

22. Zhan X, Qi J, Zhou B, Mao B. Metabolomic and transcriptomic analyses reveal the regulation of pigmentation in the purple variety of Dendrobium officinale. Sci Rep. 2020;10(1):17700

23. Ryu S, Han JH, Cho JG, Jeong JH, Lee SK, Lee HJ. High temperature at veraison inhibits anthocyanin biosynthesis in berry skins during ripening in 'Kyoho' grapevines. Plant Physiol Biochem. 2020;157:219-28.

24. Awasthi P, Gupta AP, Bedi YS, Vishwakarma RA, Gandhi SG. Mannitol stress directs flavonoid metabolism toward synthesis of flavones via differential regulation of two cytochrome P450 monooxygenases in coleus forskohlii. Front Plant Sci. 2016;7:985

25. Koo HJ, McDowell ET, Ma X, Greer KA, Kapteyn J, Xie Z, et al. Ginger and turmeric expressed sequence tags identify signature genes for rhizome identity and development and the biosynthesis of curcuminoids, gingerols and terpenoids. BMC Plant Biol. 2013;13:27.

26. Logemann $\mathrm{E}$, Hahlbrock K. Crosstalk among stress responses in plants: Pathogen defense overrides UV protection through an inversely regulated ACE/ACE type of light-responsive gene promoter unit. PNAS. 2002;99(4):2428-32.

27. Zhang YL, Fang ZZ, Ye XF, Pan SL. Identification of candidate genes involved in anthocyanin accumulation in the peel of Jaboticaba (Myrciaria cauliflora) fruits by transcriptomic analysis. Gene. 2018;676:202-13.

28. Czegeny G, Matai A, Hideg E. UV-B effects on leaves-oxidative stress and acclimation in controlled environments. Plant Sci. 2016;248:57-63.

29. Warnakulasuriya SN, Ziaullah, Rupasinghe HP. Novel long chain fatty acid derivatives of quercetin-3-O-glucoside reduce cytotoxicity induced by cigarette smoke toxicants in human fetal lung fibroblasts. Eur J Pharmacol. 2016;781:128-38.

30. Napier JA, Sayanova O. Nutritional enhancement in plants - green and greener. Curr Opin Biotechnol. 2020;61:122-7.

31. Qi T, Song $S$, Ren $Q, W u ~ D$, Huang $H$, Chen $Y$, et al. The Jasmonate-ZIMdomain proteins interact with the WD-repeat/bHLH/MYB complexes to regulate Jasmonate-mediated anthocyanin accumulation and trichome initiation in Arabidopsis thaliana. Plant Cell. 2011;23(5):1795-814.

32. Yang $\mathrm{T}$, Ma H, Zhang J, Wu T, Song T, Tian J, et al. Systematic identification of long noncoding RNAs expressed during light-induced anthocyanin accumulation in apple fruit. Plant J. 2019;100(3):572-90.

33. Gou JY, Felippes FF, Liu CJ, Weigel D, Wang JW. Negative regulation of anthocyanin biosynthesis in Arabidopsis by a miR156-targeted SPL transcription factor. Plant Cell. 2011;23(4):1512-22.

34. An JP, Yao JF, Xu RR, You CX, Wang XF, Hao YJ. Apple bZIP transcription factor MdbZIP44 regulates abscisic acid-promoted anthocyanin accumulation. Plant Cell Environ. 2018;41(11):2678-92.

35. Peng Z, Han C, Yuan L, Zhang K, Huang H, Ren C. Brassinosteroid enhances jasmonate-induced anthocyanin accumulation in Arabidopsis seedlings. J Integr Plant Biol. 2011;53(8):632-40.

36. Zhang ZZ, Li XX, Chu YN, Zhang MX, Wen YQ, Duan CQ, et al. Three types of ultraviolet irradiation differentially promote expression of shikimate pathway genes and production of anthocyanins in grape berries. Plant Physiol Biochem. 2012;57:74-83.

37. Ni J, Zhao Y, Tao R, Yin L, Gao L, Strid A, et al. Ethylene mediates the branching of the jasmonate-induced flavonoid biosynthesis pathway by suppressing anthocyanin biosynthesis in red Chinese pear fruits. Plant Biotechnol J. 2020;18(5):1223-40.

38. Agati G, Brunetti C, Di Ferdinando M, Ferrini F, Pollastri S, Tattini M. Functional roles of flavonoids in photoprotection: new evidence, lessons from the past. Plant Physiol Biochem. 2013;72:35-45.

39. Francisco RM, Regalado A, Ageorges A, Burla BJ, Bassin B, Eisenach C, et al. $A B C C 1$, an ATP binding cassette protein from grape berry, transports anthocyanidin 3-O-glucosides. Plant Cell. 2013;25(5):1840-54.

40. Mierziak J, Kostyn K, Kulma A. Flavonoids as important molecules of plant interactions with the environment. Molecules. 2014;19(10):16240-65.

41. Liu C, Jun JH, Dixon RA. MYB5 and MYB14 play pivotal roles in seed coat polymer biosynthesis in Medicago truncatula. Plant Physiol. 2014;165(4):1424-39.

42. Zhai R, Wang Z, Zhang S, Meng G, Song L, Wang Z, et al. Two MYB transcription factors regulate flavonoid biosynthesis in pear fruit (Pyrus bretschneideri Rehd.). J Exp Bot. 2016;67(5):1275-84.

43. Sun C, Deng L, Du M, Zhao J, Chen Q, Huang T, et al. A transcriptional network promotes anthocyanin biosynthesis in tomato flesh. Mol Plant. 2020;13(1):42-58

44. Pandey A, Misra P, Trivedi PK. Constitutive expression of Arabidopsis MYB transcription factor, AtMYB11, in tobacco modulates flavonoid biosynthesis in favor of flavonol accumulation. Plant Cell Rep. 2015;34(9):1515-28.

45. Anwar M, Yu W, Yao H, Zhou P, Allan AC, Zeng L. NtMYB3, an R2R3MYB from Narcissus, regulates flavonoid biosynthesis. Int J Mol Sci. 2019;20(21):5456

46. Schwinn KE, Ngo H, Kenel F, Brummell DA, Albert NW, McCallum JA, et al. The onion (Allium cepa L.) R2R3-MYB gene MYB1 regulates anthocyanin biosynthesis. Front Plant Sci. 2016;7:1865.

47. Li H, Yang Z, Zeng Q, Wang S, Luo Y, Huang Y, et al. Abnormal expression of $b H L H 3$ disrupts a flavonoid homeostasis network, causing differences in pigment composition among mulberry fruits. Hortic Res. 2020;7:83.

48. Wang XC, Wu J, Guan ML, Zhao CH, Geng P, Zhao Q. Arabidopsis MYB4 plays dual roles in flavonoid biosynthesis. Plant J. 2020;101(3):637-52.

49. Sunitha S, Loyola R, Alcalde JA, Arce-Johnson P, Matus JT, Rock CD. The role of UV-B light on small RNA activity during grapevine berry development. G3 (Bethesda). 2019:9(3):769-87.

50. Gonzalez A, Zhao M, Leavitt JM, Lloyd AM. Regulation of the anthocyanin biosynthetic pathway by the TTG1/bHLH/Myb transcriptional complex in Arabidopsis seedlings. Plant J. 2008:53(5):814-27.

51. Luo F, Cai JH, Kong XM, Zhou Q, Zhou X, Zhao YB, et al. Transcriptome profiling reveals the roles of pigment mechanisms in postharvest broccoli yellowing. Hortic Res. 2019;6:74.

52. Tamagnone L, Merida A, Parr A, Mackay S, Culianez-Macia F, Martin RC. The AmMYB308 and AmMYB330 transcription factors from antirrhinum regulate phenylpropanoid and lignin biosynthesis in transgenic tobacco. Plant Cell. 1998;10(2):135-54.

53. Ni J, Bai S, Gao L, Qian M, Zhong L, Teng Y. Identification, classification, and transcription profiles of the B-type response regulator family in pear. PLoS One. 2017:12(2):e0171523.

54. Dong T, Han R, Yu J, Zhu M, Zhang Y, Gong Y, et al. Anthocyanins accumulation and molecular analysis of correlated genes by metabolome and transcriptome in green and purple asparaguses (Asparagus officinalis, L.). Food Chem. 2019;271:18-28.

55. Yao H, Li C, Zhao H, Zhao J, Chen H, Bu T, et al. Deep sequencing of the transcriptome reveals distinct flavonoid metabolism features of black tartary buckwheat (Fagopyrum tataricum Garetn.). Prog Biophys Mol Biol. 2017;124:49-60.

56. Li W, Tan L, Zou Y, Tan X, Huang J, Chen W, et al. The effects of ultraviolet $A / B$ treatments on anthocyanin accumulation and gene expression in dark-purple tea cultivar 'Ziyan' (Camellia sinensis). Molecules. 2020;25(2):354

\section{Publisher's Note}

Springer Nature remains neutral with regard to jurisdictional claims in published maps and institutional affiliations. 